\title{
Joule-Thomson Process in the Liquefaction of Helium ${ }^{1}$
}

\author{
Edmund H. Brown and John W. Dean
}

\begin{abstract}
A dimensionless, normalized, correlating function is introduced for the specific enthalpy of helium. Using this function, the consistency of various enthalpy data is determined. These data are then used to obtain curves of helium liquefaction yield in the region of variable specific heat in terms of heat-exchanger efficiency.
\end{abstract}

\section{Introduction}

Many different devices and cycle arrangements can be used in partially cooling gas for helium liquefaction. With the exception of the Simon freeexpansion type liquefier, however-which is suitable only for small-yield, batch production-all such arrangements have a final stage at the lowest temperatures, in which a so-called Joule-Thomson process takes place. This process may be considered to include cooling of the high-pressure gas by the unliquefied return gas in a "Joule-Thomson heat exchanger", as well as cooling of the high-pressure gas by the Joule-Thomson effect, that is, isenthalpic expansion through a "Joule-Thomson valve." If the initial state of the high-pressure gas at the top of the J-T heat exchanger (which is most conveniently defined by its pressure and temperature) is fixed within a certain region, a definite mass fraction $\alpha$ of the gas passing through the J-T valve will be liquefied.

To determine the yield $\alpha$ of a liquefier, the specific enthalpies at certain points in the $\mathrm{J}-\mathrm{T}$ process must be known as functions of $P$ and $T$. The most readily available data on the specific enthalpy, $h$, of helium is from the monograph of Keesom [1], ${ }^{2}$ or the temperature-entropy diagram of Keesom published by the International Institute of Refrigeration [2]. The errors and inconsistencies of these sources below about $20^{\circ} \mathrm{K}$, however, have been pointed out in a series of papers by Zelmanov $[3,4,5,6]$. In addition, Zelmanov [6] has recalculated and redrawn both $T-s$ and $h-P$ diagrams which, as indicated later, appear to be of good accuracy and consistency. The Zelmanov $T-s$ diagram is reproduced by Zemansky [7]; and the $h-P$ diagram, by Collins [8]; who, however, also reproduces the Keesom $T-s$ diagram. Because of the crowded nature of the isobars in the region of interest for the J-T process on a $T-s$ diagram, the $h-P$ diagram is more convenient for liquefier calculations.

At the beginning of this study there was no easy way to judge the relative value of various conflicting sets of data. In order to determine, at least, the consistency of such data, a new dimensionless, normalized correlating function $w(P, T)$ was introduced. With this function, the consistency of Zelmanov's work was evident, and both the lack of self-consistency of the $T-s$ diagrams of Keesom, and

This work was supported by the Department of the Navy, Bureau of Aeronautics, Helium Branch.

2 Figures in brackets indicate the literature references at the end of this paper. the inconsistency of these diagrams in certain regions with Keesom's own density data was apparent. In addition, it appears that there may be some value in this new function $w(P, T)$ in theoretical studies, since it implies that, above certain pressures, the enthalpy of a real gas may be approximately given by the sum of two linearly independent functions, one of the pressure alone, the other, of the temperature alone. However, not enough time was available in this study for further investigations in this direction.

A second factor which complicates the design of helium liquefiers is the behavior of the specific heat $c_{p}$ of the real gas at low temperatures. At high temperatures $c_{p}$ of a gas gradually increases with increasing pressure; but, at a lower temperature, dependent on the pressure, this trend is reversed. Ignorance of this effect has occasionally led the unwary to design liquefiers in which the temperature difference $\Delta T$ at the bottom of the J-T heat exchanger would have to be reversed. This phenomenon, which has been hinted at by Keesom and discussed qualitatively by Zelmanov, is investigated further in this study. In order to determine performance, a method of analyzing $\mathrm{J}-\mathrm{T}$ heat exchangers in terms of one parameter - the heat exchanger efficiency $\eta$-is presented, and graphs of yield curves for helium based on this method are included.

The purpose of this paper, then, consists of three things: First, and most important, to affirm the work of Zelmanov (an adaptation of Zelmanov's $h-P$ diagram is included as figure 1); second, to present the correlating function $w(P, T)$ and discuss some of its properties; and third, to show quantitatively the effect of the variation of $c_{p}$ in the $\mathrm{J}$-T heat-exchanger on the liquefied fraction $\alpha$.

\section{Correlating Function $w(P, T)$}

The enthalpy of a gas can be expressed as

$$
h(P, T)=\int^{T} c_{p}(P, T) d T+G(P)
$$

where $G$ is a constant of integration. For an ideal gas, $c_{p}(0, T)=c_{p}^{\circ}$; and, for helium, which is a monatomic gas, $c_{p}^{\circ}=(5 R / 2)=5.193 \mathrm{j} / \mathrm{g}^{\circ}{ }^{\circ} \mathrm{K}$. In defining the enthalpy of the ideal gas, $h^{\circ}=h(0, T)$, the constant of integration $G(0)$ was equated with the latent heat of evaporation at $0^{\circ} \mathrm{K}, \mathrm{L}^{\circ}$, which is equivalent to fixing the enthalpy (which at this point is equal to the internal energy) of the liquid at $P=0, T=0$ as zero. According to Swenson [9], the extrapolated 
value of $L^{\circ}$ is $14.95 \mathrm{j} / \mathrm{g}$. Thus, the expression used for helium in the ideal gas state is

$$
h^{\circ}=c_{p}^{\circ} T+L^{\circ}=5.193 T+14.95 \mathrm{j} / \mathrm{g} \text {. }
$$

Just as an enthalpy in an "ideal gas" state can be defined by extrapolating to zero pressure, so an enthalpy in an "ideal condensed phase" state, $h_{0}=h(P, 0)$, can be defined by extrapolating to zero temperature. If $V(P, 0)$ is the specific volume at zero temperature, then

$$
\left(\frac{\partial h_{0}}{\partial P}\right)_{T=0}=V(P, 0)=V(0,0) e^{-\int_{0}^{p} k(P, 0) d P} .
$$

An attempt was made to express the extrapolated isothermal compressibility at $0^{\circ} \mathrm{K}, k(P, 0)=-(1 / V)$ $[(\partial V) /(\partial P)]_{T}$, by an empirical equation which would have led to an expression for $h_{0}$ as a function of exponential integrals, $\mathrm{Ei}(k)$. However, since this required some simplifying assumptions, was not particularly suited for engineering calculations, and lacked theoretical justification, $h_{0}$ was finally determined by extrapolating Keesom's density data [1] with the aid of expansivity and compressibility data of Atkins and Edwards [10]. The resulting curves for $h_{0}, V(P, 0)$, and also $k(P, 0)$, are included as figures 2,3 , and 4 . It should be mentioned that Swenson's extrapolated value [9] of $V(0,0)$, for the liquid seems to be in error.

Having, now, both $h^{\circ}=h(0, T)$ and $h_{0}=h(P, 0)$, the remaining "anomalies" of both the real gas and the condensed phases can all be contained in a function $w(P, T)$ defined by

$$
w(P, T)=\frac{h(P, T)-h_{0}(P)}{h^{\circ}(T)}
$$

which is not only dimensionless, as is evident from the definition, but has a total range of zero to one; zero, for the condensed phases as $T \rightarrow 0^{\circ} \mathrm{K}$; one, for the gas as $T \rightarrow \infty$; one, for the gas as $P \rightarrow 0$; and zero, for the condensed phase as $P \rightarrow \infty$.

Here, we use the symbols $T \rightarrow \infty$ or $P \rightarrow \infty$ merely for convenience to indicate relatively high and increasing temperature and pressure, but not so high that the character of the "elementary" particles implied by the choice of $L^{\circ}$ and $h^{\circ}(T)$ is destroyed. Thus, we assume, for instance, that the pressure is not so high as to force an electron from the atomic shell, converting the solid into metallic helium (estimated required pressure 1,000,000 atm), or that the temperature is so high as to form a gas mixture of ionized particles. This restriction would be of even greater importance for diatomic gases, such as hydrogen, which begin to dissociate from molecular to atomic particles at relatively low temperatures. Presumably, all the properties of the $w$ function could be reobtained in such cases by a redefinition of $L^{\circ}$ and $h^{\circ}(T)$. For example, $h^{\circ}(T)$ would, then, have to be the enthalpy of the gas in the atomic ideal gas state and $L^{\circ}$ the difference between the energy of isolated atoms at $0^{\circ} \mathrm{K}$ and the energy of (for hydrogen) the crystal at $0^{\circ} \mathrm{K}$ and $0 \mathrm{~atm}$. Some of these effects, such as dissociation, however, are so large that the introduction of a more general $w$ function would result in characteristics in the regions of interest for cryogenic processes too small to be useful.

From the theoretical point of view this function does have the disadvantage that a discontinuity in $[(\partial w) /(\partial P)]_{T}$ - though not in $w$-is introduced by the liquid-solid transformation for $h_{0}$ at $25 \mathrm{~atm}$. However, this change in slope of $w$ is slight, being given approximately by $\Delta[(\partial h) /(\partial P)]_{T}=0.0524 \mathrm{j} / \mathrm{g}-$ atm. (from the data of Swenson [9]). It would probably be of considerable interest to compare curves of $w\left(P_{r}, T_{r}\right)$ as functions of reduced temperature $T_{r}$ and pressure $P_{r}$ for several gases.

Further insight into the characteristic behavior of $w(P, T)$ can be obtained by examination of the graphs (at end of paper). From figure 6 which gives $w$ as a function of $P$ with $T$ as a parameter, it can be seen that all isotherms start with the same value, $w=1, P=0$; that outside of the liquid II region, all isotherms are everywhere decreasing functions of $P$; that the isotherms are continuous except on passing through phase changes; that no two isotherms cross; that the slopes of all isotherms approach zero with increasing pressure; and that, although the total percent change in $w$ along an isotherm is greater the smaller the temperature, the pressure at which the slope of an isotherm becomes effectively zero increases with increasing temperature (for the $0^{\circ} \mathrm{K}$ isotherm the slope becomes zero at zero atmosphere, the value of $w$ changing discontinuously from one to zero through the vapor dome, and then remaining zero for all nonzero pressures).

From figure 7 , giving $w$ as a function of $T$ with $P$ as a parameter a similar set of statements can be made; all isobars start with the same value, $w=0$ $T=0$; all isobars are everywhere increasing functions of $T$; the isobars are continuous except on passing through phase changes; outside of the liquid II region no two isobars cross; the slopes of all isobars outside the liquid II region approach zero with increasing temperature, and approach zero more rapidly the lower the pressure (the slope of the zero atmosphere isobar being zero for all nonzero temperatures); the value of $w$ for all isobars approaches one with increasing temperature, and approaches more rapidly the lower the pressure (the zero atmosphere isobar changing discontinuously from zero to one at zero atmosphere). The liquid II region sketched on this diagram is somewhat uncertain, due principally to lack of time for more extended calculations in an area of little interest for liquefier design.

The limiting behavior of $w(p, T)$ can also be determined by considering its definition. It is obvious that for $T \rightarrow 0, p \neq 0, w \rightarrow 0$; for $p \rightarrow 0, T \neq 0, w \rightarrow 1$; while for $T \rightarrow 0, p=0$, or $p \rightarrow 0, T=0, w$ becomes undefined, but lies within the range 0 to 1 . This is the region of liquid-vapor coexistence at $0^{\circ} \mathrm{K}$ and zero atmospheres.

When $T$ increases without limit at constant and finite $p$,

$$
\lim _{T \rightarrow \infty} w(p, T)=\frac{\lim _{T \rightarrow \infty} h(p, T)}{\lim _{T \rightarrow \infty} h^{\circ}(T)}=1 .
$$


The second equality of this equation may be obtained on the assumption that no matter how high the pressure the system will become a "gas" for a sufficiently large increase in temperature, with enthalpy $h$ approaching the enthalpy of an ideal gas $h^{\circ}$. This may be seen for a region in which a virial equation of state applies, where the higher virial type coefficients in the pressure series are sometimes taken as decreasing approximately as increasing power of $1 / T$.

For increasing pressure, however,

$$
\begin{aligned}
\lim _{p \rightarrow \infty} w(p, T)=\lim _{p \rightarrow \infty} \frac{1}{h^{\circ}} \int_{0}^{T} c_{p}(p, T) d T \\
=\frac{1}{h^{\circ}} \int_{0}^{T} \lim _{p \rightarrow \infty} c_{p}(p, T) d T=0
\end{aligned}
$$

since $\lim _{p \rightarrow \infty} c_{p}(p, T) \rightarrow 0$, as can be deduced from the fact that the slopes of the isobars with fixed $T$ on a temperature entropy diagram, $[(\partial T) /(\partial S)]_{p}=\left(T / c_{p}\right)$, approach infinity with increasing pressure (recall, however, our restriction on the meaning of $P \rightarrow \infty$ ).

The above combination of well-defined behavior and range of magnitude makes the checking and cross checking of enthalpy data with $w-P$ and $w-T$ diagrams relatively easy. In particular, it has indicated many errors and inconsistencies in the $T-s$ diagrams of Keeson, and affirmed the consistency of the $T-s$ and $h-P$ diagrams of Zelmanov.

Since the enthalpy of helium can be expressed as

$$
h(P, T)=h_{0}(P)+w(P, T) h^{\circ}(T),
$$

the fact that the isotherms on the $w-P$ diagram shown in figure 6 become approximately horizontal at pressures that are not extremely high is of some theoretical interest; for this behavior of the isotherms is equivalent to stating that $w(P, T)$ becomes practically a function of temperature, alone, even for large pressure ranges; thus, since $h^{\circ}$ is also a function of the temperature, alone, the enthalpy can be expressed approximately as the sum of a function of the pressure $h_{0}$ (which might be considered a strain energy term), and a function of the temperature $w(T) h^{\circ}(T)$ (which might be considered a thermal energy term).

\section{Liquefier Performance and Heat- Exchanger Efficiency}

Before discussing the J-T heat exchanger for helium use, it is desirable to review a few general relations. Heat exchanger efficiency is defined as the ration of actual heat flow to the ideal heat flow, $\eta=\dot{Q}_{\text {actual }} / \dot{Q}_{\text {ideal }}$, where the ideal is that which would occur if the product of the total effective heat exchange area and the over-all coefficient of heat transfer, $A K$, is made infinite. However, in obtaining $\eta, \dot{Q}_{\text {actual }}$ and $\dot{Q}_{\text {ideal }}$ must be evaluated for the same conditions; that is, the flows, pressures, and inlet temperatures of both streams must be held constant. Thus, for an arbitrarily defined heat exchanger, the efficiency will be an explicit function of the flows, and - if the fluid properties are not constant - an implicit function of temperatures and pressures as well.

The efficiency of a two-stream, counter-flow, heat exchanger can be expressed analytically in three different forms, depending on the flow conditions. If $\dot{m}_{1}, \dot{m}_{2}$ are the mass flows, $c_{p 1}, c_{p 2}$, the specific heats, $W_{1}=-\dot{m}_{1} c_{p 1}, W_{2}=\dot{m}_{2} c_{p 2}$, the heat capacity flows in the two streams, and writing, for convenience, $(1 \cdot / W)=\left[\left(1 / W_{1}\right)+\left(1 / W_{2}\right)\right]$, the expressions for efficiency are

$$
\begin{gathered}
\eta=\frac{1-e^{-A\left(\frac{\bar{K}}{W}\right)}}{1+\frac{\bar{W}_{2}}{\bar{W}_{1}} e^{-A\left(\frac{\bar{K}}{\bar{W}}\right)}, W>0,} \\
\eta=\frac{1-e^{+A\left(\frac{\bar{K}}{\bar{W}}\right)}}{1+\frac{\bar{W}_{1}}{\bar{W}_{2}} e^{+A\left(\frac{\bar{K}}{W}\right)}, W<0} \\
\left.\eta=\frac{1}{1+\left(\frac{\bar{W}_{2}}{W}\right)\left(\frac{\bar{W}}{A K}\right)}=\frac{1-\left(\frac{\bar{W}_{1}}{\bar{W}^{\prime}}\right)\left(\frac{\bar{W}}{A K}\right)}{\bar{W}^{2}}\right)
\end{gathered}
$$

where bars above quantities indicate that some kind of integrated average over the heat exchanger must be taken. However, obtaining such averages requires a knowledge of the temperature distributions in the streams, and if these were known, there would be no problem in the first place. Thus, for heat exchangers with variable fluid properties, the above formulas can only be used to obtain approximations for efficiencies.

It is our purpose, however, to show how liquefier performance depends on an arbitrarily assigned efficiency, rather than analyzing how the efficiency of a given heat exchanger may be determined. Thus, our results will be correct for any exchanger that has the assigned efficiency under the assumed flow conditions. Since the efficiency of a given exchanger varies with the flow, an important question is, to what extent may a curve of constant efficiency also be considered a curve of performance with fixed exchanger characteristics? To this we can only give a qualitative answer; since the efficiencies of J-T heat exchangers are ordinarily very high, even with equal heat capacity flows in the streams, the efficiency will not be a very strong function of the flows. To justify this statement, we use an example, based on an approximation of constant and equal fluid properties in the streams of a typical counter-flow heat exchanger.

Remembering that the film coefficients are given by equations of the form $\mathrm{Nu}=\mathrm{C} \operatorname{Re}^{0.8} \operatorname{Pr}^{n}$, writing $\dot{K}_{0}$ for the over-all coefficient of transfer for the particular case where equal flow exists and $\sigma_{0}=$ $\left.\left[\left(A K_{0}\right) / W_{2}\right)\right]$ and introducing the liquefied fraction $\alpha$ to specify the relation between the mass flows. 
$W_{1}=-(1-\alpha) W_{2}$, the efficiency is given by the equation

$$
\frac{1}{\eta}=1-\frac{\alpha}{1-\exp \frac{2 \sigma_{0} \alpha}{1-\alpha+(1-\alpha)^{0.2}}}, 0 \leq \alpha \leq 1
$$

or, in the limit as $\alpha \rightarrow 0$, as evaluated by l'Hospital's Rule,

$$
\frac{1}{\eta}=1+\frac{1}{\sigma_{0}}, \alpha=0
$$

From these it is easy to see that, for any $\alpha, \eta$ increases with increasing $\sigma_{0}$; and, for any $\sigma_{0}, \eta$ increases with $\alpha$. However, in the limit, as $\sigma_{0} \rightarrow \infty$, the limiting curve $\eta(\alpha)$ becomes merely the horizontal line $\eta=1$. For large, but finite $\sigma_{0}, \eta(\alpha)$ will remain nearly horizontal and nearly straight, so that - as mentioned before $-\eta$ is not a very strong function of $\alpha$ for J-T heat exchangers.

To get an idea of the magnitude of the change we assume some arbitrary values. Suppose $K_{0}=50$ w $/ \mathrm{ft}^{2}{ }^{\circ} \mathrm{K}$ and $A=25 \mathrm{ft}^{2}$. Also, let $\dot{m}_{2}=0.01 \mathrm{~kg} / \mathrm{sec}$, $c_{p 2}=5000 \mathrm{j} / \mathrm{kg}^{\circ}{ }^{\circ} \mathrm{K}$, so that $W_{2}=50 \mathrm{w} /{ }^{\circ} \mathrm{K}$, and $\sigma_{0}=25$. Substitution of these values in the above equations shows that the efficiency varies from 96 percent at $\alpha=0$, to 100 percent at $\alpha=1$. In real liquefiers the variation would be even less, since $\alpha$ would ordinarily be in the range 0.2 to 0.5 .

\section{Calculation of Performance From Efficiency}

The liquefaction rate $\alpha$ will now be determined from the inlet, high-pressure stream pressure and temperature and the heat exchanger efficiency using only specific enthalpies from an $h-P$ diagram. In making an enthalpy balance, the specific enthalpy $h_{i}$ at the inlet of the high-pressure stream is determined by the assumed pressure and temperature, and the specific enthalpy $h_{0}$ at the inlet of the low-pressure stream is assumed to be that of the saturated vapor at 1 atm. Thus, only two quantities, the specific enthalpy $h$, above the J-T valve, and the specific enthalpy $h_{r}$ of the return gas at the outlet of the lowpressure stream., are available, and only one of these is independent since

$$
h_{i}-h_{\jmath}=(1-\alpha)\left(h_{r}-h_{g}\right) .
$$

Furthermore, if $h_{j}^{\prime}$ and $h_{r}^{\prime}$ are the values of $h_{j}$ and $h_{r}$ when $\eta=100$ percent, the efficiency in any other case is given by

$$
\eta=\frac{h_{\tau}-h_{g}}{h_{\tau}^{\prime}-h_{g}}=\frac{h_{i}-h_{j}}{h_{i}-h_{j}^{\prime}}
$$

so that we can write

and

$$
h_{j}=h_{i}-\eta\left(h_{i}-h_{j}^{\prime}\right)
$$

$$
h_{r}=h_{g}+\eta\left(h_{r}-h_{r g}^{\prime}\right) .
$$

It should be reemphasized here that the efficiency is defined in terms of two heat flows (or enthalpy changes) under the same conditions of inlet pressures and temperatures and the same mass flows.

Since enthalpies must balance for the whole J-T process, as well as just the heat-exchanger, we can also write

$$
h_{\imath}=\alpha h_{L}+(1-\alpha) h_{r}
$$

where $h_{L}$ is the specific enthalpy of the saturated liquid at one atmosphere. Thus $\alpha$ can be expressed by either of the equations

$$
\alpha=\frac{h_{r}-h_{i}}{h_{r}-h_{L}}=\frac{h_{g}-h_{\jmath}}{h_{g}-h_{L}} .
$$

Substituting for $h_{r}$ and $h_{j}$ their expressions in terms of $h_{r}^{\prime}$ and $h_{j}^{\prime}$, and writing $L_{v}=h_{g}-h_{L}$, these equations become

$$
\alpha=\frac{\eta-\frac{h_{i}-h_{g}}{h_{r}^{\prime}-h_{g}}}{\eta+\frac{L_{v}}{h_{r}^{\prime}-h_{g}}}=\eta \frac{h_{\imath}-h_{j}^{\prime}}{L_{v}}-\frac{h_{i}-h_{g}}{L_{v}} .
$$

Now, a reasonable assumption is that, for $\eta=100$ percent the temperature difference between streams $\Delta T$ becomes zero at some point in the heat exchanger. We should not naively assume that, in the most general case, this might not be in the middle of the exchanger, since this is exactly what would happen if $c_{p}$ for the high-pressure stream were lower than that for the low-pressure stream at the higher temperatures, and conversely at lower temperatures. For helium in the J-T process range, however, the reverse is the case, and thus, $\Delta T$ must diminish towards the top of the exchanger, and - for suitable initial pressure and temperature diminish again towards the bottom of the exchanger. Thus, the ideal case will be characterized by having $\Delta T=0$ at either the top or the bottom of the exchanger. If we write $h_{r}^{\prime \prime}$ for the value of $h_{r}^{\prime}$ when $\Delta T=0$ at the top, and $h_{j}^{\prime \prime}$ for the value of $h_{j}^{\prime}$ when $\Delta T=0$ at the bottom, then - since both $h_{r}^{\prime \prime}$ and $h_{j}^{\prime \prime}$ can be determined immediately from an $h-P$ diagram - there will be two different possible equations for the liquefaction rate $\alpha$ in which all the quantities are known:

$$
\begin{gathered}
\text { (a) } \alpha=\frac{\eta-\frac{h_{i}-h_{g}}{h_{r}^{\prime \prime}-h_{g}}}{\eta+\frac{L_{v}}{h_{r}^{\prime \prime}-h_{g}}}, \Delta T \rightarrow 0 \text { at the top; } \\
\text { (b) } \alpha=\eta \frac{h_{i}-h_{j}^{\prime \prime}}{L_{v}}-\frac{h_{i}-h_{g}}{L_{v}}, \Delta T \rightarrow 0 \text { at the bottom. }
\end{gathered}
$$

In case (a) we can determine $h_{j}^{\prime}$ by an enthalpy balance and see whether $h_{i}^{\prime}>h_{j}^{\prime \prime}$; if not, the assumption $\Delta T=0$ at the top must be wrong, and we would have to change to case (b). Similarly, in case (b) we can determine $h_{r}^{\prime}$ and check that $h_{r}^{\prime}<h_{r}^{\prime \prime}$. Actually, however, such a check is unnecessary, since the two assumptions will result in two curves inter- 
secting where $\Delta T=0$ simultaneously at the top and bottom of the exchanger, and it will be immediately evident which branch should be used to form the composite curve for the liquefaction rate.

Such composite curves of $\alpha$ as a function of $P$ with $\eta$ as a parameter, for helium, are shown in figures 9,10 , and 11 . The discontinuities in the slopes of these curves would be disturbing if one did not recall that, even for the case of constant fluid properties, the efficiency of an exchanger is expressed by two different formulas, depending on whether $W$ is positive or negative. It is just the change of the total average value of $W$ from positive to negative with the variation of specific heat of helium, which produces two independent branches in the curves shown.

The differing way $\alpha$ varies with $\eta$ for these two branches should be especially noted. For lower pressures, relatively large changes in $\eta$ have only a small effect on $\alpha$. If, however, a pressure is chosen at or slightly above the "optimum." pressure, $\alpha$ will not only decrease rapidly with increasing pressure, but at a given pressure, will drop drastically with slight reductions in heat-exchanger efficiency. 'Therefore, it is desirable to be somewhat conservative in the design pressure for a liquefier in order to allow for possibly too optimistic estimates of heat-exchanger performance.

\section{References}

[1] W. H. Keesom, Helium (Elsevier, Amsterdam, 1940).

[2] International Institute of Refrigeration, T-S diagram for helium (Paris, 1941).

[3] J. L. Zelmanov, On some peculiarities observed in the liquefaction of helium, Compt. rend. acad. sci. USSR 19, 469 (1938).

[4] J. L. Zelmanov, On the liquefaction of helium by means of the Joule-Thomson effect, Compt. rend. acad. sci. USSR 20, 537 (1938).

[5] J. L. Zelmanov, A new method of helium liquefaction by means of the Joule-Thomson effect, Compt. rend. acad. sci. USSR 22,25 (1939).

[6] J. L. Zelmanov, The entropy diagram for helium at low temperatures, J. Phys. USSR 8, 135 (1944).

[7] M. W. Zemansky, Heat and thermodynamies (MeGrawHill Book Co., Inc., New York, N. Y., 1951).

[8] S. C. Collins, Helium liquefiers and carriers, Handbuch der physik, 14, 112 (Springer, Berlin, 1956).

[9] C. A. Swenson, The liquid-solid transformation in helium near absolute zero, Phys. Rev. $\mathbf{7 9 ,} 626$ (1950).

[10] K. R. Atkins and M. H. Edwards, Coefficient of expansion of liquid helium II, Phys. Rev. 97, 1429 (1955).

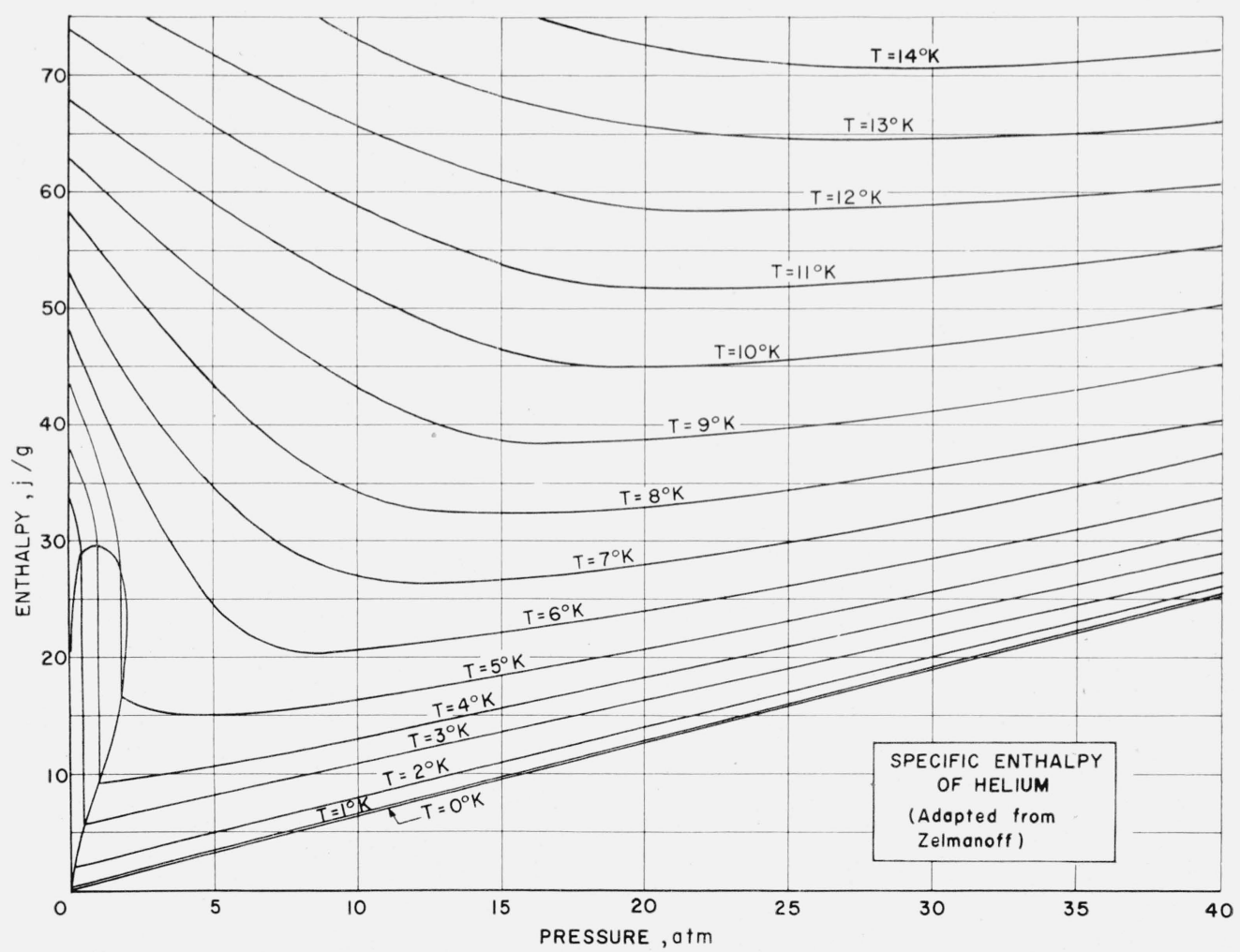

FIGURE 1. Specific enthalpy of helium.

Adapted from Zelmanov 


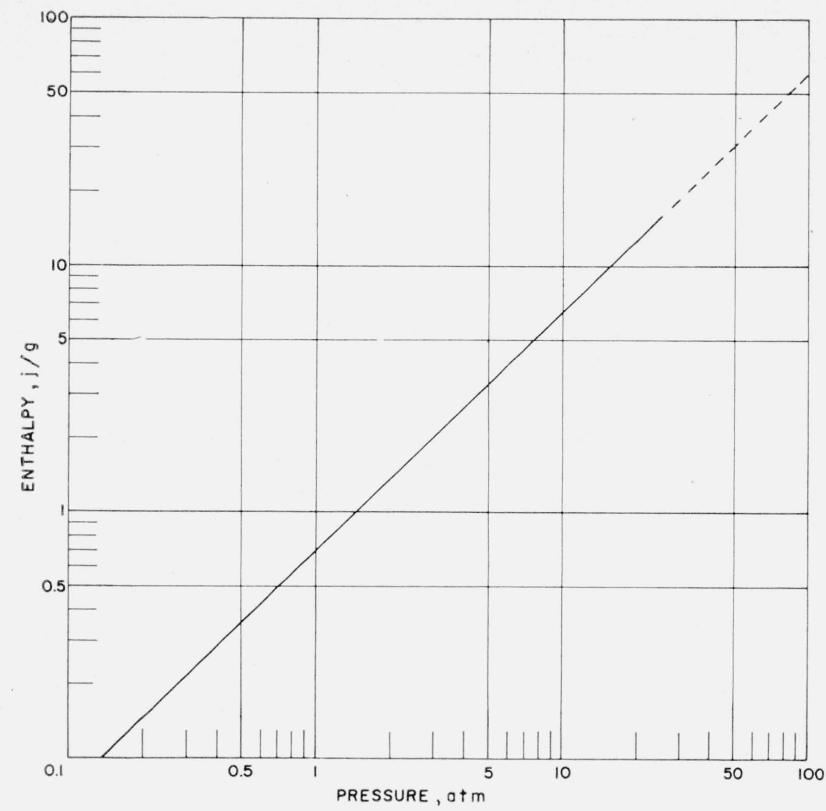

FIGURE 2. Specific enthalpy of helium at $0^{\circ} K, h_{\mathrm{o}}(p)$ versus pressure.

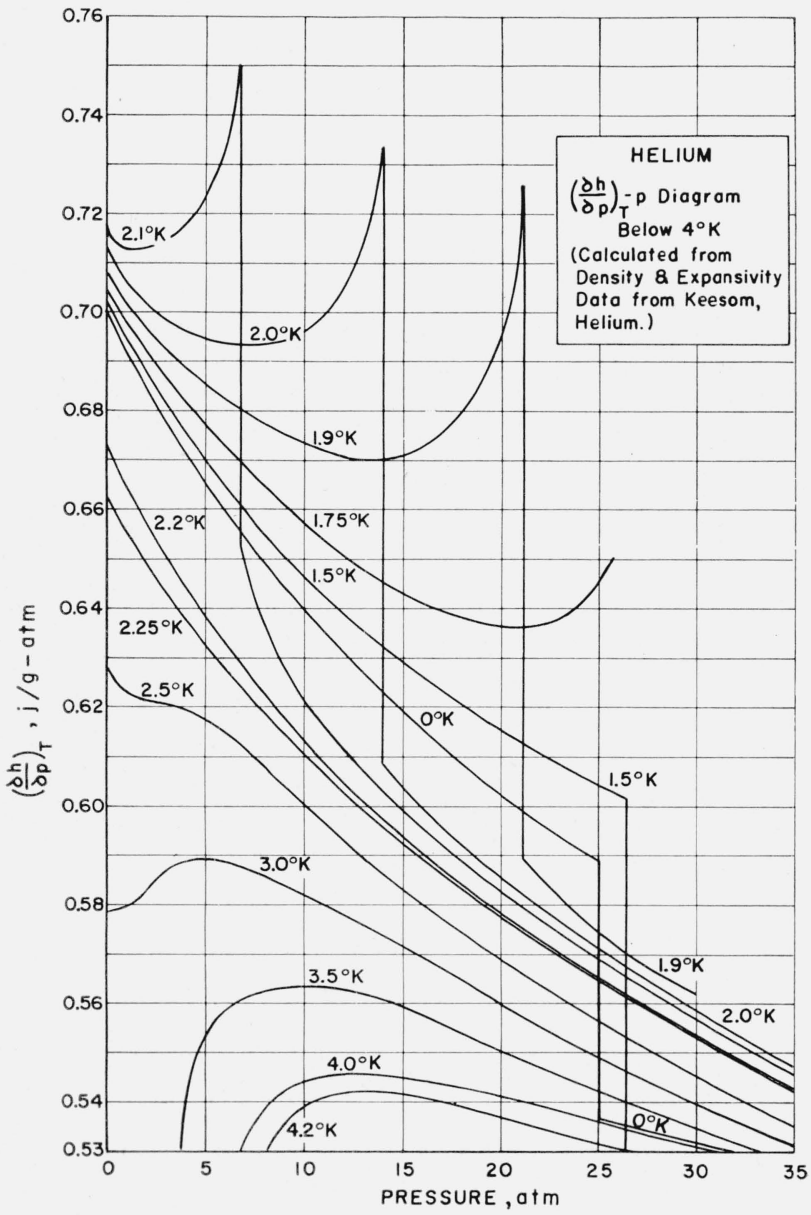

FiguRE 5. $[(\partial h) /(\partial p)]_{T}$ versus pressure of helium below $4^{\circ} \mathrm{K}$. Calculated from density and expansivity data from Keesom, helium.

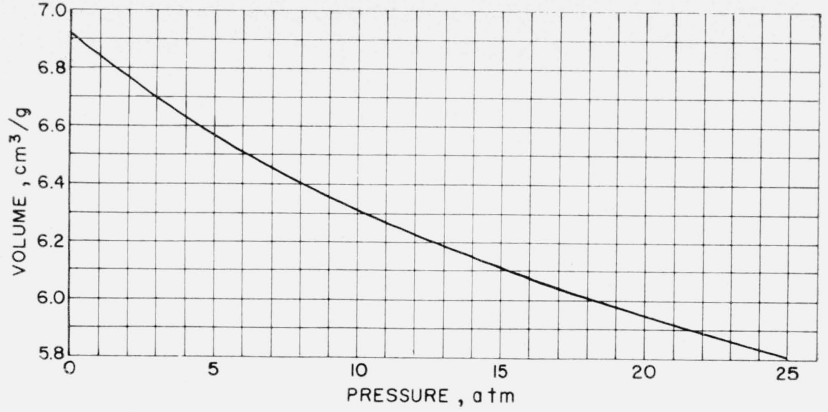

Figure 3. Specific volume of helium at $0^{\circ} \mathrm{K}$.

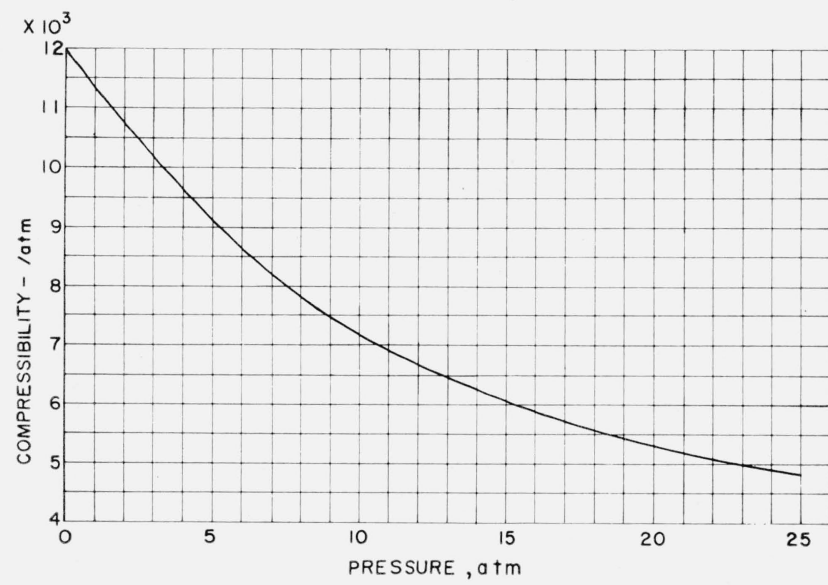

Figure 4. Compressibility of helium at $0^{\circ} \mathrm{K}$.

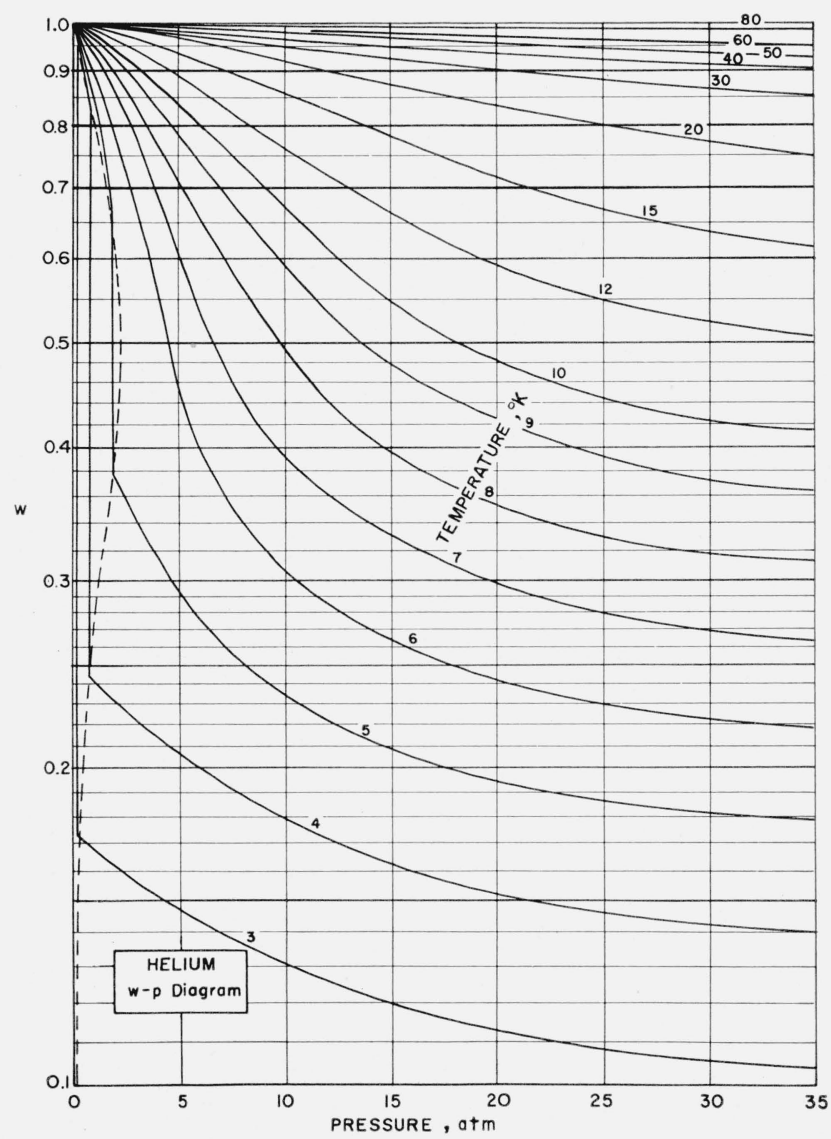

Figure 6. Helium $w$-p diagram. 


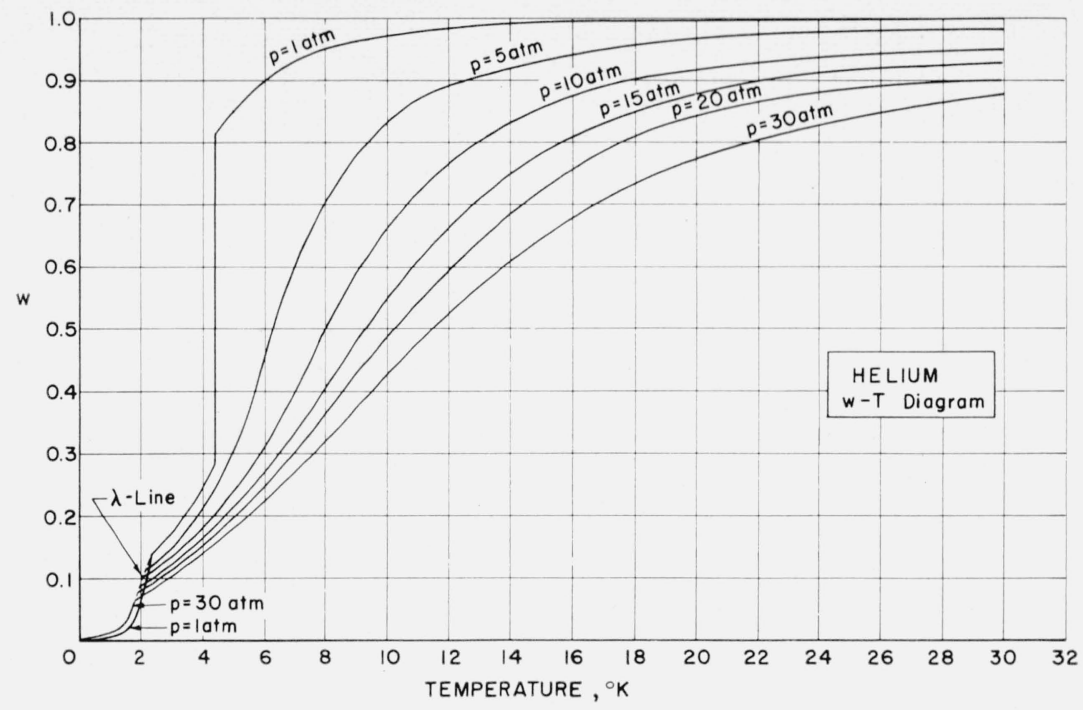

Figure 7. Helium $w$-T diagram.

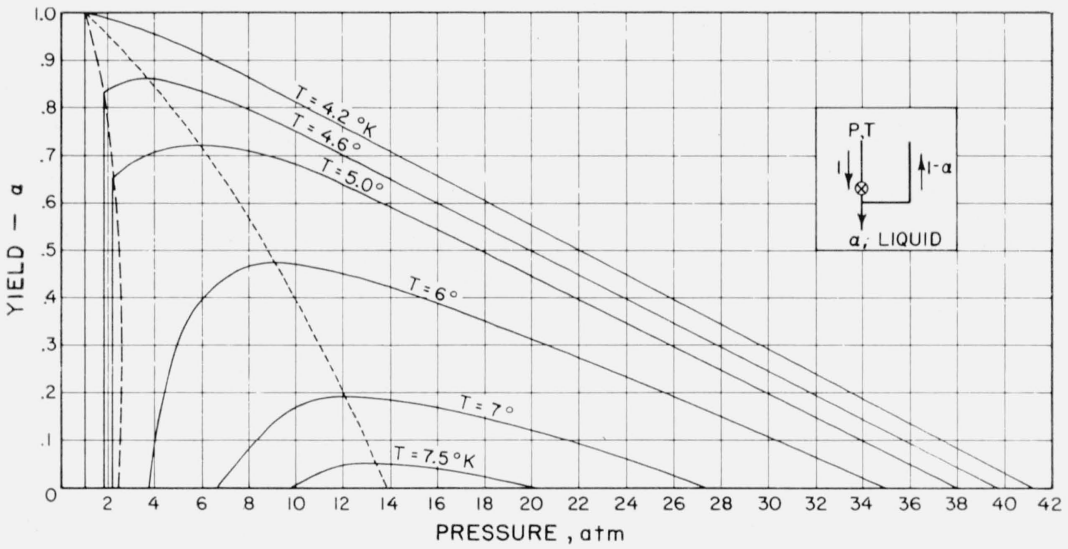

FIgURE 8. Yield $\alpha$ as a function of pressure and temperature at the $J-T$ valve.

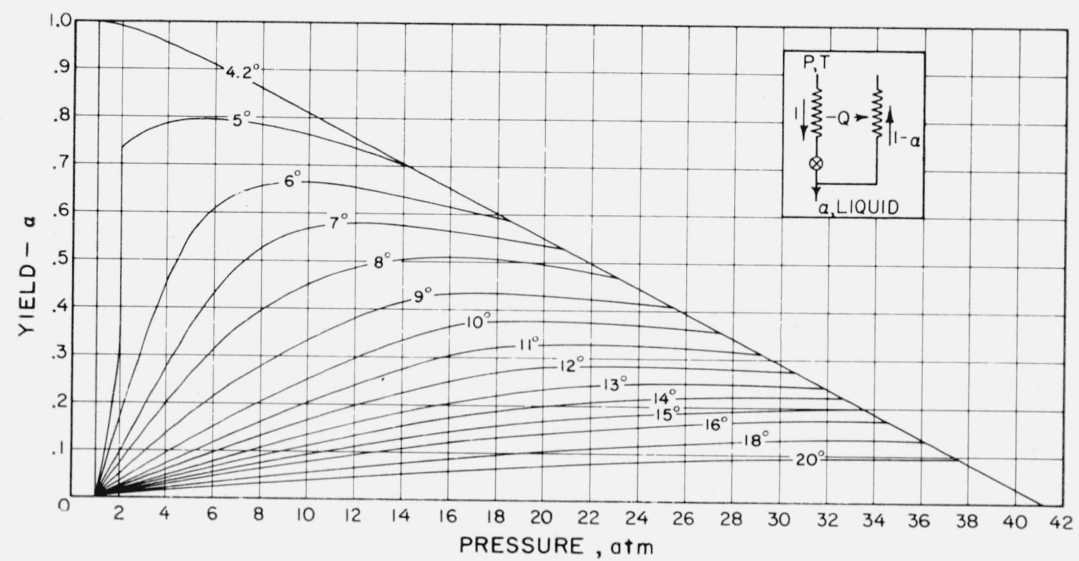

FIgURE 9. Yield $\alpha$ with $J$-T heat exchanger efficiencies $\eta$ equals 100 percent as a function of HP inlet temperature and pressure. 


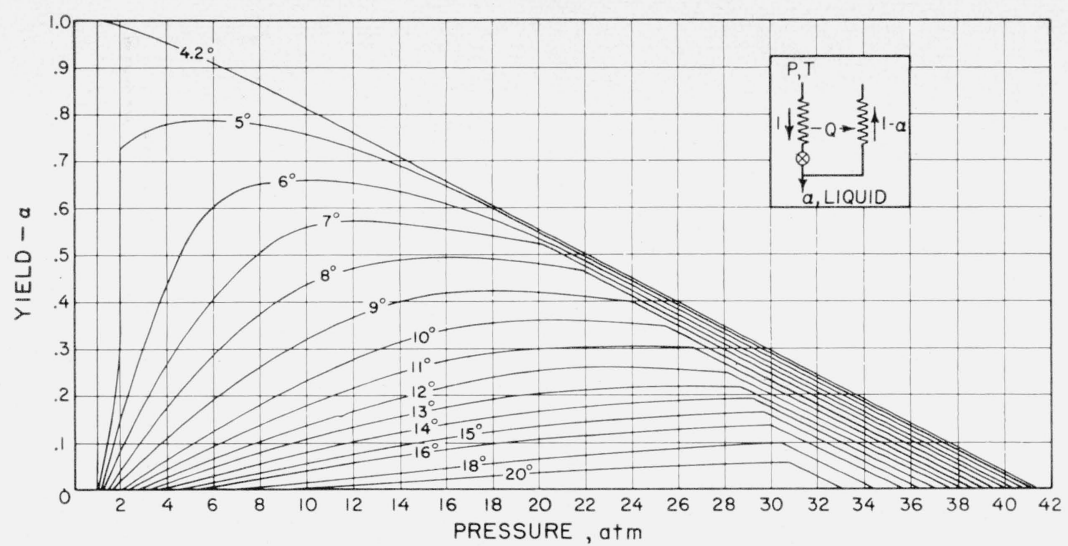

FIgURE 10. Yield $\alpha$ with $J$-T heat exchanger efficiency $\eta$ equals 95 percent as a function of $H P$ inlet pressure and temperature.

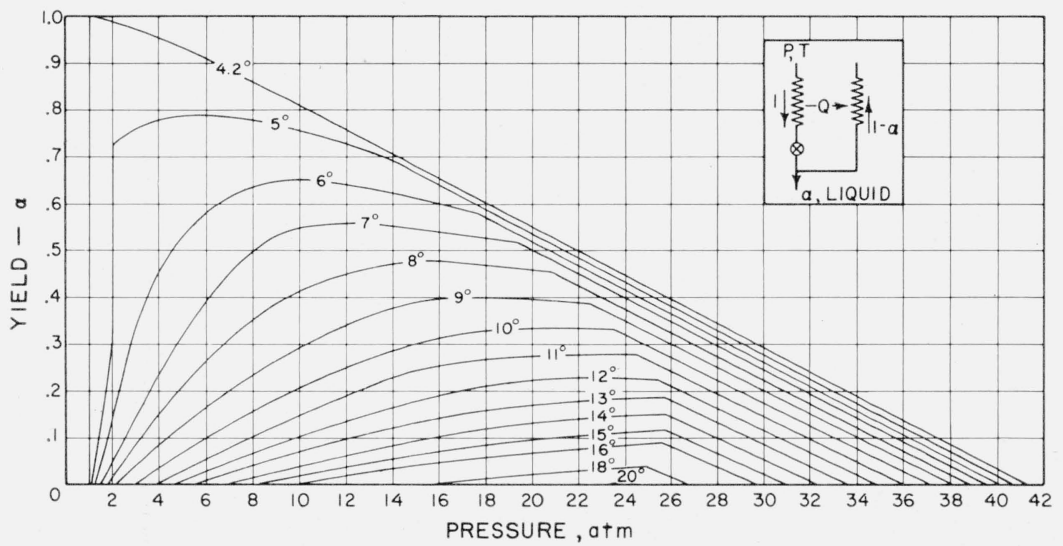

FIGURE 11 Yield $\alpha$ with $J-T$ heat exchanger efficiency $\eta$ equals 90 percent as a function of HP inlet pressure and temperature.

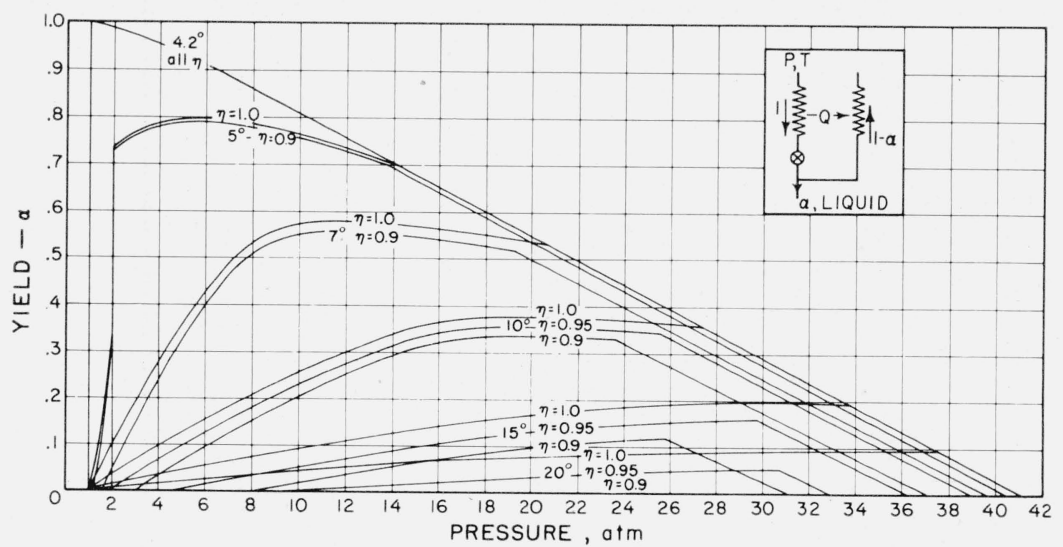

FIGURE 12. Yield $\alpha$ for several heat exchanger efficiencies as a function of inlet temperature and pressure.

Boulder, Colo., August 30, 1957. 